\title{
TRATAMENTO DAS FRATURAS DO TERÇO DISTAL DA TÍBIA - FIXAR OU NÃO A FÍBULA?
}

\author{
TREATMENT OF THE DISTAL FRACTURES OF THE TIBIA - SHALL WE FIX THE FIBULA?
}

Pedro José Labronici'; Rolix Hoffmann²; José Sergio Franco3; Paulo Roberto Barbosa de Toledo Lourenço4; Hélio Jorge Alvachian Fernandes ${ }^{5}$ Fernando Baldy dos Reis ${ }^{6}$

\section{RESUMO}

Objetivo: Comparar os resultados da fixação ou não da fíbula no tratamento das fraturas do terço distal da tíbia, com haste intramedular e placa em ponte. Métodos: Foram 47 fraturas em 47 pacientes, sendo que em 21 pacientes foi utilizada a haste intramedular bloqueada não fresada e em 26 a placa em ponte (placa de compressão dinâmica larga ou estreita) pela técnica minimamente invasiva. Todas as fraturas da fíbula se encontravam no mesmo nível ou abaixo da fratura da tíbia. Resultados: No grupo tratado com fixação da fíbula, a média do tempo de consolidação foi de 14,6 semanas. No grupo tratado sem fixação da fíbula, a média do tempo de consolidação foi de 14,3 semanas. No grupo de pacientes tratados com fixação da fíbula observou-se uma proporção de desvio angular em varo $(6,3 \%)$ significativamente menor que o subgrupo sem fixação de fíbula $(32,3 \%)$, e com desvio angular em valgo $(62,5 \%)$ significativamente maior que o grupo sem fixação de fíbula $(32,3 \%)$. Conclusão: Os benefícios da fixação da fíbula permanecem ainda controversos quando ocorrem fraturas associadas com a tíbia. Em relação à consolidação, não houve diferença significativa entre os grupos.

Descritores: Fratura da Tibia; Fratura da Fibula; Fixação Interna

\section{ABSTRACT}

Objective: To compare the results of fibula fixation (or not fixation) in the treatment of fractures located in the distal third part of the tibia, by using intramedullary nailing and bridge plate. Method: 47 fractures in 47 patients were studied. Twenty-one patients were treated with non-reammed, interlocking intramedullary nailing, and 26 patients were treated with wide or narrow dynamic compressional plates (using a minimally invasive technique). All of the fibula fractures are located at the same level or below the tibia fractures. Results: in the group of patients treated with fibula fixation, the average healing time was 14.6 weeks. In the group of patients treated without fibula fixation, the average healing time was 14.3 weeks. In the group of patients treated with fibula fixation it was observed a significantly smaller proportion of valgus angular deviation (6.3\%) than in the group of patients treated without fibula fixation (32.3\%). Conclusions: The benefits of fibula fixation still keeping controversial when tibial fractures are associates. Regarding fracture healing, there was no significant difference between the studied fracture groups.

Keywords: Tibial Fracture; Fibular Fracture; Internal Fixation

1 - Doutor em Medicina pela Universidade Federal de São Paulo - Escola Paulista de Medicina e Chefe de Clínica do Serviço de Ortopedia e Traumatologia Prof. Dr. Donato D’Ângelo - Hospital Santa Teresa, Petrópolis/RJ, Brasil.

2 - Médico do Serviço de Ortopedia e Traumatologia Prof. Dr. Donato D’Ângelo - Hospital Santa Teresa, Petrópolis/RJ, Brasil.

3 - Chefe do Departamento e Professor Adjunto de Ortopedia e Traumatologia da Faculdade de Medicina da UFRJ/RJ, Brasil.

4 - Médico Responsável pelo Grupo de Trauma do Hospital de Ipanema, Rio de Janeiro, Brasil.

5 - Professor Adjunto Doutor em Medicina - Médico do Setor de Traumatologia do Aparelho Locomotor da Disciplina de Traumatologia do Departamento de Ortopedia e Traumatologia da UNIFESP/EPM, São Paulo, Brasil.

6 - Professor Livre Docente e Chefe do Setor de Traumatologia do Aparelho Locomotor da Disciplina de Traumatologia do Departamento de Ortopedia e Traumatologia da UNIFESP/EPM, São Paulo, Brasil.

Trabalho realizado no Serviço de Ortopedia e Traumatologia Prof. Dr. Donato D'Ângelo, Hospital Santa Teresa, Petrópolis, RJ, Brasil.

Endereço para correspondência: Av. Roberto Silveira, 187 apto 601 - 25685-040 - Petrópolis, RJ. E-mail: plabronici@globo.com

Trabalho recebido para publicação: 15/03/11, aceito para publicação: 15/04/11. 


\section{INTRODUÇÃO}

O tratamento da fratura do terço distal da tíbia continua sendo um grande desafio. São fraturas onde é difícil obter um alinhamento adequado e, portanto, lesões que colocam em risco a distribuição de cargas sobre o tornozelo. A redução é ainda mais difícil quando existe fratura da fíbula no mesmo nível da tíbia. Este padrão de fratura reflete um mecanismo de trauma de alta energia e causa aumento da instabilidade angular, rotacional, encurtamento do membro e maior lesões das partes moles ${ }^{(1,2)}$.

Existem, na literatura, vários tipos de tratamento para as fraturas do terço distal da tíbia ${ }^{(3-8)}$. Porém, as duas técnicas mais utilizadas são: a haste intramedular bloqueada e a placa colocada de maneira minimamente invasiva.

O impacto clínico da fixação da fíbula como coadjuvante no tratamento das fraturas do terço distal da tíbia tratadas com haste intramedular ou placa permanece desconhecido. Alguns autores acreditam que a fixação da fíbula ajudaria a reduzir o alinhamento rotacional e sagital o que pode ser difícil de obter com a haste intramedular isolada. Quando se utiliza a placa na tíbia, a fixação da fíbula ajudaria a restaurar o comprimento e as deformidades angulares e rotacionais, reduzindo assim, o risco de consolidação viciosa ${ }^{(1,2,8,18)}$.

O objetivo deste trabalho foi comparar os resultados da fixação ou não da fíbula no tratamento das fraturas do terço distal da tíbia, com haste intramedular e placa.

\section{MÉTODOS}

No período entre 1997 a 2005, foram tratados 203 pacientes com fraturas fechadas ou expostas dos graus I, II e IIIA da diáfise da tíbia no Hospital Santa Teresa, Petrópolis. Em caráter retrospectivo e utilizando a classificação AO das fraturas, selecionamos para este estudo 47 pacientes portadores de fraturas do terço distal da tíbia. Foram 47 fraturas em 47 pacientes, sendo que em 21 pacientes foi utilizada a haste intramedular bloqueada não fresada $\left(\right.$ Baumer $^{\circledR}$ e haste universal $\mathrm{AO}^{\circledR}$ ) e em 26 a placa (placa de compressão dinâmica larga ou estreita) pela técnica minimamente invasiva. Em ambas as técnicas, os dados obtidos incluíam aspectos demográficos tais como: idade, gênero e tabagismo. Foram, também, computados dados referentes a detalhes da lesão tais como: mecanismo do trauma determinante, lesões associadas, tipo de fratura de acordo com a classificação $\mathrm{AO}^{(19)}$ e fixação ou não da fíbula. Os grupos foram comparados de acordo com a fixação ou não da fíbula. Para interpretação dos resultados funcionais levou-se em

Rev Bras Ortop. 2011;46(Suppl 1):23-7 consideração a ocorrência de desvio angular residual e a taxa de consolidação nos grupos. Os desvios angulares foram classificados por Helfet et $a l^{(12)} \mathrm{em}:<5^{\circ}$ de varo, $<10^{\circ}$ de valgo e $<10^{\circ}$ de antecurvato/retrocurvato.

Com o objetivo de verificar se existe relação significativa entre as variáveis com a fixação da fíbula (presente e ausente) foram aplicados os seguintes métodos: para comparação de dados quantitativos (numéricos) foi utilizado o teste de Mann-Whitney (não paramétrico) e para comparações de proporções (dados qualitativos) foi aplicado o teste de qui-quadrado $\left(\mathbf{C}^{2}\right)$ ou o exato de Fisher.

Foi utilizado método não-paramétrico, pois algumas variáveis não apresentaram distribuição normal (distribuição Gaussiana) devido a dispersão dos dados e falta de simetria da distribuição. O critério de determinação de significância adotado foi o nível de 5\%.

\section{RESULTADOS}

Nos pacientes tratados com haste intramedular, a idade variou entre 17 e 57 anos, com média de 33 anos. Nos pacientes tratados com placa, a idade variou entre 14 e 90 anos, com média de 36 anos.

No grupo de pacientes que foram tratados com fixação da fíbula, 10 eram do sexo masculino e seis do feminino, sendo que sete apresentaram fratura do lado direito e nove à esquerda. Segundo a classificação $\mathrm{AO}^{(19)}$ das fraturas, três pacientes apresentavam fratura do tipo A, 11 do tipo B e dois do tipo C. As fraturas expostas foram classificadas pelo método de Gustilo et $a l^{(20)} \mathrm{e}$ somente um paciente apresentava fratura exposta do grau IIIA. As fraturas fechadas foram classificadas pelo método de Oestern et al ${ }^{(21)}$ : cinco pacientes apresentaram fratura do tipo 0 , oito do tipo 1 , dois do tipo 2 . Não houve pacientes com fratura do tipo 3 .

No grupo de pacientes que foram tratados sem fixação da fíbula, 27 eram do sexo masculino e quatro do feminino, sendo que 15 apresentaram fratura do lado direito e 16 à esquerda. Segundo a classificação $\mathrm{AO}^{(19)}$, das fraturas, 14 pacientes apresentavam fratura do tipo A, 15 do tipo B e dois do tipo C. As fraturas expostas foram classificadas pelo método de Gustilo et $a l^{(20)}$ : sete pacientes apresentaram fratura exposta do grau I e sete do grau II. As fraturas fechadas foram classificadas pelo método de Oestern et al $^{(21)}$ : sete pacientes apresentaram fratura do tipo 0, oito do tipo 1 e dois do tipo 2. Em ambos os grupos a fratura da fíbula se encontrava ao mesmo nível ou abaixo da fratura da tíbia (Tabela 1). 
Tabela 1 - Análise descritiva geral das características qualitativas.

\begin{tabular}{c|c|c|c}
\hline Variável & Categoria & $\mathbf{n}$ & $\%$ \\
\hline Sexo & masc & 37 & 78,7 \\
\hline & fem & 10 & 21,3 \\
\hline Lado & direito & 22 & 46,8 \\
\hline Causa do acidente & esquerdo & 25 & 53,2 \\
\hline & atropelamento & 12 & 25,5 \\
\hline & moto & 16 & 34,0 \\
\hline & queda altura & 13 & 27,7 \\
\hline & trauma direto & 3 & 6,4 \\
\hline Tipo de fratura & ac. auto & 2 & 4,3 \\
\hline & queda escada & 1 & 2,1 \\
\hline
\end{tabular}

\begin{tabular}{c|c|c|c}
\hline Fratura fechada & 0 & 12 & 37,5 \\
\hline & 1 & 16 & 50 \\
\hline Fratura exposta & 2 & 4 & 12,5 \\
\hline & II & 7 & 46,7 \\
\hline Fumante & IIla & 1 & 46,7 \\
\hline & sim & 22 & 6,7 \\
\hline Traumas associados & não & 25 & 46,8 \\
\hline Fonte: Hospital Santa Teresa, Serviço do Prof. Dr. Donato D'Ângelo, Petrópolis.
\end{tabular}

No grupo tratado com fixação da fíbula, o tempo médio de seguimento foi de 22,6 meses variando entre seis e 48 meses. A média do tempo de consolidação foi de 14,6 semanas variando entre oito e 56 semanas. No grupo tratado sem fixação da fíbula, a média do tempo de seguimento foi de 46,8 meses variando entre 10 e 112 meses. A média do tempo de consolidação foi de 14,3 semanas variando entre sete e 56 semanas. Portanto, o tempo de consolidação foi similar em ambos os grupos. A tabela 2 fornece a média, desvio padrão (DP), mediana, mínimo e máximo das variáveis numéricas segundo a fixação da fíbula, e o correspondente nível descritivo do teste estatístico (valor $p$ ). A análise estatística foi realizada pelo teste de Mann-Whitney, para as variáveis idade, tempo entre o acidente e a cirurgia, tempo de seguimento e tempo de consolidação.

Verificamos se existe diferença significativa entre as variáveis dos grupos com e sem fixação da fíbula. No grupo de pacientes tratados com fixação da fíbula foi observada uma proporção de fratura fechada $(93,8 \%)$ significativamente maior que o grupo sem fixação de fíbula $(54,8 \%)$, com $p=0,007$ (Figura 1).

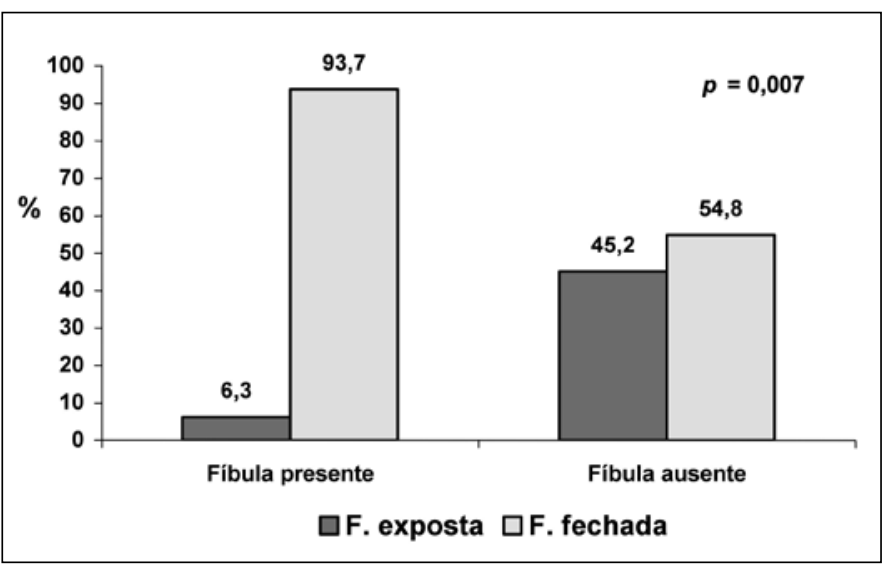

Figura 1 - Fixação da fíbula versus tipo de fratura.

Fonte: Hospital Santa Teresa, Serviço do Prof. Dr. Donato D’Ângelo, Petrópolis.

O grupo de pacientes tratados com fixação da fíbula teve sua fratura tibial predominantemente fixada com placa $(75 \%)$. Isto foi estatisticamente significativo quando comparado ao grupo onde a fíbula não foi fixada, em que a indicação de placa não ocorreu na maioria dos casos. $(45,2 \%)$, com $p=0,05$ (Figura 2).

Tabela 2 - Análise estatística das variáveis numéricas segundo a fixação da fíbula.

\begin{tabular}{|c|c|c|c|c|c|c|c|c|}
\hline Variável & Fíbula & $\mathbf{n}$ & Média & DP & Mediana & Mínimo & Máximo & $p$ valor \\
\hline \multirow[t]{2}{*}{ Idade (anos) } & $\operatorname{sim}$ & 16 & 41,1 & 18,1 & 36,5 & 18 & 90 & \multirow{2}{*}{0,089} \\
\hline & não & 31 & 32,1 & 12,5 & 31 & 14 & 57 & \\
\hline \multirow[t]{2}{*}{ Tempo acidente-cirurgia (horas) } & $\operatorname{sim}$ & 16 & 67,8 & 70,9 & 60 & 3 & 240 & \multirow{2}{*}{0,29} \\
\hline & não & 31 & 47,6 & 74,5 & 24 & 3 & 360 & \\
\hline \multirow[t]{2}{*}{ Tempo de seguimento (meses) } & $\operatorname{sim}$ & 16 & 22,6 & 16,5 & 20 & 6 & 48 & \multirow{2}{*}{0,0008} \\
\hline & não & 31 & 46,8 & 27,4 & 36 & 10 & 112 & \\
\hline \multirow[t]{2}{*}{ Tempo de consolidação (sem) } & $\operatorname{sim}$ & 16 & 14,6 & 11,4 & 12 & 8 & 56 & \multirow{2}{*}{0,54} \\
\hline & não & 31 & 14,3 & 9,0 & 12 & 7 & 56 & \\
\hline
\end{tabular}




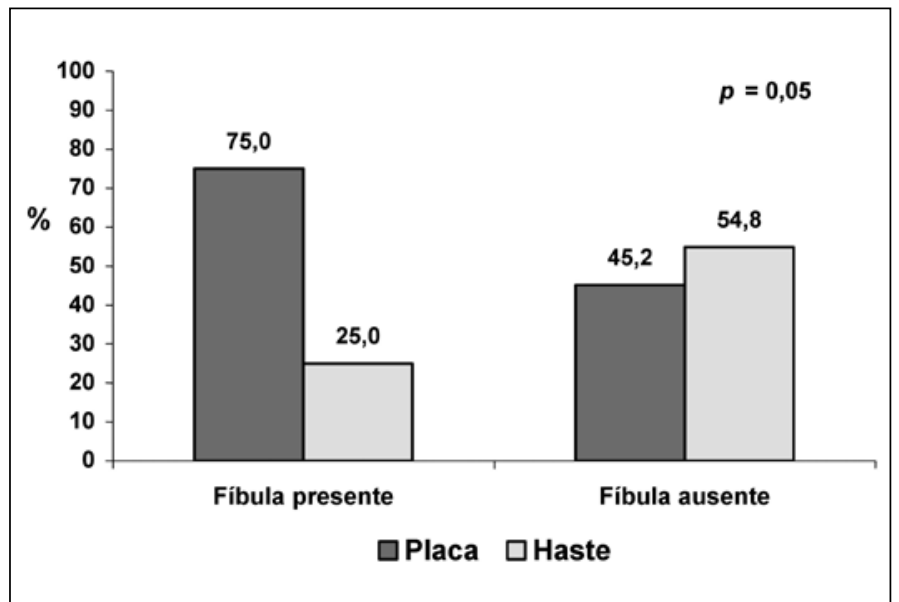

Figura 2 - Fixação da fíbula versus tipo de osteossíntese. Fonte: Hospital Santa Teresa, Serviço do Prof. Dr. Donato D’Ângelo, Petrópolis.

No grupo de pacientes tratados com fixação da fíbula observou-se uma proporção de desvio angular em varo $(6,3 \%)$ significativamente menor que o subgrupo sem fixação de fíbula $(32,3 \%), \operatorname{com} p=0,045$, e com desvio angular em valgo $(62,5 \%)$ significativamente maior que o grupo sem fixação de fíbula $(32,3 \%)$, com $p=0,047$ (Figuras 3 e 4).

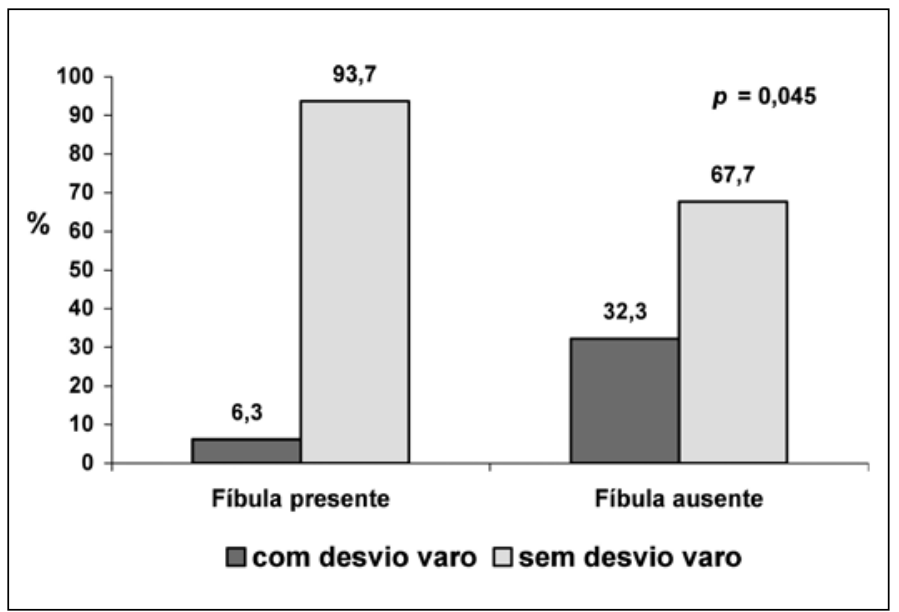

Figura 3 - Fixação da fíbula versus desvio angular varo.

Fonte: Hospital Santa Teresa, Serviço do Prof. Dr. Donato D’Ângelo, Petrópolis.

\section{DISCUSSÃO}

Estudos biomecânicos em cadáver demonstraram que a fixação da fratura da fíbula associada com a fratura distal da tíbia tratada com haste intramedular bloqueada reduz os desvios angulares e a consolidação viciosa. Também, ajuda a evitar o "efeito deslizamento do pára-brisa" (windshield wiper) entre o parafuso de bloqueio e o furo da haste, que favorece movimen-

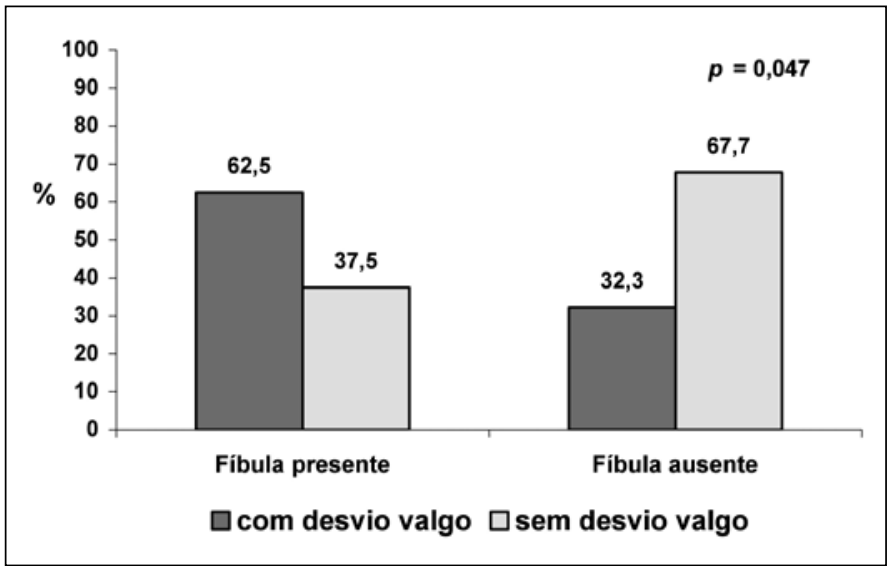

Figura 4 - Fixação da fíbula versus desvio angular valgo. Fonte: Hospital Santa Teresa, Serviço do Prof. Dr. Donato D’Ângelo, Petrópolis.

tos em varo-valgo pelo parafuso ${ }^{(1)}$. Mosheiff et al ${ }^{(8)}$ e Tyllianakis et $a l^{(18)}$ tratando com fixação da fíbula, relataram uma baixa tendência de deformidades da tíbia, por acreditarem ser fácil o alinhamento do membro durante a colocação da haste. Dogra et al ${ }^{(26)}$ relataram que em três pacientes dos 15 casos de sua série apresentaram angulação em varo ou valgo $>$ que $5^{\circ}$, sem fixação da fíbula. Schmidt et $a l^{(27)}$ relataram que a fixação da fíbula deve ser realizada antes da fixação da tíbia com haste intramedular quando existe grande desvio da fíbula, pois ajuda a restaurar o alinhamento do membro ou quando há uma instabilidade do tálus. Entretanto, acreditam que a fixação da fíbula contribui para o aumento da morbidade. Goldsztajn et al ${ }^{(28)}$ trataram 26 pacientes com haste intramedular fresada e encontraram $88,5 \%$ de redução anatômica da tíbia, no pós-operatório imediato, sem a necessidade de fixação da fratura da fíbula.

A fixação da fratura da fíbula associada com a fratura do terço distal da tíbia tratada com placa pela técnica minimamente invasiva deve ser avaliada individualmente, pois não foram estabelecidas ainda as indicações precisas ${ }^{(2)}$. Bedi et $a l^{(2)}$, apesar de não fixarem rotineiramente a fíbula, demonstraram que esta técnica ajuda a restaurar o comprimento do membro corrigindo as deformidades angulares e rotacionais nas fraturas da fíbula com grande desvios ou cominuição, portanto, reduzindo o risco de consolidação viciosa. Em nosso estudo, as fraturas tratadas com fixação da fíbula associada a fratura da tíbia tratadas com haste intramedular auxiliou a restabelecer o comprimento, desvios angulares (valgo) e a rotação do membro principalmente nas fraturas do tipo $\mathrm{B}$ e $\mathrm{C}$ da classificação AO, mesmo quando utilizamos a técnica de Schmitt 
et $a l^{(29)}$, que utiliza um fio de Kirschner paralelo a articulação do tornozelo. Com este fio de referência, além de ajudar na redução da fratura da tíbia orienta a precisa colocação da haste, que deve ser colocada com um ângulo reto com este fio. Quando utilizamos a placa, a fixação da fíbula além de auxiliar na restauração do comprimento do membro e na correção das deformidades angulares e rotacionais, manteve boa anatomia do membro e facilitou na pré-moldagem da placa, ajudando na redução da fratura e evitando a deformidade mais comum em valgo. Esta deformidade foi significantemente maior nos pacientes que não foram tratados com fixação da fíbula tanto com as hastes intramedulares como com placas.

\section{REFERÊNCIAS}

1. Kumar A, Charlebois SJ, Cain EL, Smith RA, Daniels AU, Crates JM. Effect of fibular plate fixation on rotational stability of simulated distal tibial fractures treated with intramedullary nailing. J Bone Joint Surg [Am] 2003; 85:604-8.

2. Bedi A, Le TT, Karunakar MA. Surgical treatment of nonarticular distal tibia fractures. J Am Acad Orthop Surg 2006; 14:406-16.

3. Robinson CM, McLauchlan GJ, McLean IP, Court-Brown CM. Distal metaphyseal fractures of the tibia with minimal involvement of the ankle: Classification and treatment by locked intramedullary nailing. J Bone Joint Surg [Br] 1995; 77: 781-787.

4. Gorczyca JT, McKale J, Pugh K, Pienkowski D. Modified tibial nails for treating distal tibia fractures. J Orthop Trauma 2002; 16: 18-22.

5. Trafton PG: Tibial shaft fractures, in Browner BD, Levine AM, Jupiter JB (eds): Skeletal Trauma, ed 3. Philadelphia, PA: WB Saunders, 2003, vol 2, p 2131-256.

6. Sarmiento A, Latta LL: 450 closed fractures of the distal third of the tibia treated with a functional brace. Clin Orthop Relat Res 2004; 428:261-71.

7. Bourne RB: Pylon fractures of the distal tibia. Clin Orthop Relat Res 1989; 240:42-6.

8. Mosheiff R, Safran O, Segal D, Liebergall M. The unreamed tibial nail in the treatment of distal metaphyseal fractures. Injury 1999; 30:83-90.

9. Konrath G, Moed BR, Watson JT, Kaneshiro S, Karges DE, Cramer KE. Intramedullary nailing of unstable diaphyseal fractures of the tibia with distal intraarticular involvement. J Orthop Trauma 1997; 11:200-5.

10. Tornetta III P, Casey D, Creevy WR. Nailing proximal and distal tibia fractures. Rosemont, IL: Orthopaedic Trauma Association. Final Program \& Membership Directory. 2000: 131-132.

11. Richter D, Ostermann PA, Ekkernkamp A, Hahn MP, Muhr G. Distal tibial fracture: An indication for osteosynthesis with an unreamed intramedullary nail? [German] Langenbecks Arch Chir Suppl Kongressbd 1997; 114:1259-61.

12. Helfet DL, Shonnard PY, Levine D, Borrelli J Jr. Minimally invasive plate osteosynthesis of distal fractures of the tibia. Injury 1997; 28(suppl 1): A42-A47.

13. Oh CW, Kyung HS, Park IH, Kim PT, Ihn JC. Distal tibia metaphyseal fractures treated by percutaneous plate osteosynthesis. Clin Orthop Relat Res 2003; 408:286-91

14. Francois J, Vandeputte G, Verheyden F, Nelen G. Percutaneous plate fixation of fractures of the distal tibia. Acta Orthop Belg 2004; 70:148-54.
Nossos resultados também reforçaram o conceito que a fixação da fíbula associada ao tratamento da fratura do terço distal da tíbia não demonstrou nenhum efeito sobre a consolidação óssea, conforme a literatura ${ }^{(26,27)}$.

\section{CONCLUSÃO}

Os benefícios da fixação da fíbula associadamente à fixação de fraturas do terço distal da tíbia permanecem controversos. Em nosso estudo a fixação da fíbula não agregou diferença nas taxas de consolidação da fratura tibial. Estudos prospectivos randomizados, onde se adote um metodo único para tratamento das fraturas tibiais devem ser desenhados para avaliar o efeito da fixação da fíbula na fixação de fraturas do terço distal da tíbia.

15. Borg T, Larsson S, Lindsjo U. Percutaneous plating of distal tibial fractures: Preliminary results in 21 patients. Injury 2004; 35:608-14.

16. Maffulli N, Toms AD, McMurtie A, Oliva F. Percutaneous plating of distal tibial fractures. Int Orthop 2004; 28:159-62.

17. Khoury A, Liebergall M, London E, Mosheiff R. Percutaneous plating of distal tibial fractures. Foot Ankle Int 2002; 23:818-24.

18. Tyllianakis $M$, Megas $P$, Giannikas $D$, Lambiris $E$. Interlocking intramedullary nailing in distal tibial fractures. Orthopedics 2000; 23:805-8.

19. Müller ME, Allgöwer M, Schneider R, Willenegger H. Manual de Osteossíntese: Técnicas Recomendadas pelos Grupos AO-ASIF. $3^{a}$ ed. São Paulo: Manole, 1993. p.151-8. Tradução: Nelson Gomes de Oliveira.

20. Gustilo RB, Anderson JT. Prevention of infection in the treatment of one thousand and twenty-five open fractures of long bones: retrospective and prospective analyses. J Bone Joint Surg [Am] 1976; 58:453-8.

21. Oestern HJ, Tscherne H. Pathophysiology and classification of soft tissue injuries associated with fractures, in Tscherne $H$, Gotzen $L$ (eds): Fractures with soft tissue injuries. Berlin, Germany, Springer-Verlag, 1984, p 1-9.

22. Thordarson DB. Complications after treatment of tibial pilon fractures: prevention and management strategies. J Am Acad Orthop Surg 2000; 8:253-65.

23. Bonar SK, Marsh JL. Tibial plafond fractures: changingprinciples of treatment. J Am Acad Orthop Surg 1994; 2:247-54.

24. Farouk O, Krettek C, Miclau T, Schandelmaier P, Guy P, Tscherne H. Minimally invasive plate osteosynthesis and vascularity: Preliminary results of a cadaver injection study. Injury 1997; 28(suppl 1): A7-A12.

25. Krettek C. Concepts of minimally invasive plate osteosynthesis. Injury 1997; 28(suppl 1): S-A1-S-A2.

26. Dogra AS, Ruiz AL, Thompson NS, Nolan PC. Dia-metaphyseal distal tibial fractures - treatment with a shortened intramedullary nail: a review of 15 cases. Injury 2000; 31:799-804.

27. Schmidt AH, Finkemeier CG, Tornetta III P. Treatment of closed tibial fractures. Instructional Course Lectures, the American Academy of Orthopaedic Surgeons. J Bone Joint Surg [Am] 2003; 85:352-68.

28. Goldsztajn F, Guimarães JM, Rocha TH, Correa M, Dias MV, Lemgruber L. Fraturas do terço distal dos ossos da perna: É necessário fixar a fíbula? Congresso Brasileiro de Trauma. Santos, SP, 2007.

29. Schmitt AK, Nork SE, Winquist RA. Intramedullary nailing of distal metaphyseal tibial fractures. Read at the Annual Meeting of the Orthopaedic Trauma Association 2000; 13: San Antonio, Tx. 